$5-20-2010$

Dynamics of electron wave propagation in photoionization microscopy. II. Quantum-mechanical formulation

L. B. Zhao

Follow this and additional works at: https://scholarworks.wm.edu/aspubs

Part of the Physics Commons 


\title{
Dynamics of electron wave propagation in photoionization microscopy. II. Quantum-mechanical formulation
}

\author{
L. B. Zhao and J. B. Delos \\ Department of Physics, College of William \& Mary, Williamsburg, Virginia 23187, USA
}

(Received 8 April 2010; published 20 May 2010)

\begin{abstract}
Complementary to the semiclassical method developed in the preceding paper, here we develop a quantummechanical approach to characterize propagation of electron waves to large distances in photoionization microscopy of hydrogen atoms in uniform external electric fields. A formula for outgoing electron waves from the atomic source produced in photoionization has been derived. Spatial distributions of electron probability densities can be determined using the obtained formula. The electron waves that propagate to a large distance due to photoionization from the $n=2$ initial state of hydrogen atoms in electric fields has been calculated and the resulting probability densities are presented. Comparison with semiclassical results is made, and they are found to be in good agreement. A detailed analysis is given of strong quantum tunneling effects found in our calculations.
\end{abstract}

DOI: 10.1103/PhysRevA.81.053418

PACS number(s): $32.80 . \mathrm{Fb}, 03.65 . \mathrm{Sq}, 07.81 .+\mathrm{a}$

\section{INTRODUCTION}

This is the second of the two papers about the dynamics of electron wave propagation in the combined Coulomb and uniform external electric fields. In the first paper [1], a semiclassical open-orbit theory has been presented. This theory, based on an assumption that electron waves propagate along classical motion paths, provides a clear and intuitive physical picture (see Fig. 1 therein) to interpret structures of observed geometrical interference patterns in photoionization microscopy. With the help of an analysis from quantummechanical calculations, open-orbit theory predicted a strong quantum tunneling effect in photoionization microscopy. In this paper, we develop a quantum-mechanical approach, with which quantum-mechanical calculations were performed in Ref. [1], to compute spatial distributions of electron wave functions, or more strictly square moduli.

The problem of $\mathrm{H}$ atoms in the presence of uniform external electric fields has been investigated by many authors (see, e.g., Landau and Lifshitz's book [2]). Several methods of numerical integrations of the Schrödinger equations were developed, such as the power-series expansion method of Damburg and Kolosov [3], the Numerov method of Luc-Koenig and Bachelier [4], and the renormalized Numerov method of Alijah et al. [5]. The positions and widths of resonances and photoionization cross sections were published and found to be in excellent agreement with experiment. However, to our knowledge, no quantum-mechanical calculations have been reported predicting spatial distributions of electron probability densities for $\mathrm{H}$ atoms in electric fields. Such a theoretical investigation becomes indispensable with the development of experimental technology in photoionization microscopy, and therefore it is the aim of the present paper.

This paper is organized as follows. In Sec. II, we derive the expression for outgoing wave functions. In Sec. III, numerical solutions of the Schrödinger equation are outlined in mixed parabolic and semiparabolic coordinates. In Sec. IV, spatial distributions of probability densities of electron wave propagation, produced by photoionization of hydrogen atoms from the $n=2$ initial states in electric fields, are presented and compared with results from calculations of open-orbit theory. Section V summaries the approach and the main conclusion.

\section{FORMULATION OF OUTGOING WAVES}

Let us consider $\mathrm{H}$ atoms placed in a uniform external electric field and assume that the electric field is directed along the $z$ axis. The Hamiltonian for this system is, in atomic units,

$$
\widehat{H}=-\frac{1}{2} \nabla^{2}-\frac{1}{r}+\mathscr{F} z
$$

where $\mathscr{F}$ denotes the electric field strength measured in $F_{0}=$ $m^{2} e^{5} / \hbar^{4} \approx 5.142 \times 10^{9} \mathrm{~V} / \mathrm{cm}$. The Schrödinger equation is separable in parabolic coordinates [6]. Here for convenience of numerical computations, we adopt mixed parabolic and semiparabolic coordinates, suggested by Alijah et al. [5]:

$$
\begin{gathered}
\xi=\sqrt{r+z}, \\
\eta=r-z .
\end{gathered}
$$

The energy eigenfunctions of the Schrödinger equation may be written as

$$
\Psi(\xi, \eta, \phi)=\frac{u(\xi)}{\sqrt{\xi}} \frac{v(\eta)}{\sqrt{\eta}} \frac{e^{i m \phi}}{\sqrt{2 \pi}},
$$

where $u(\xi)$ and $v(\eta)$ satisfy the ordinary differential equations

$$
\begin{gathered}
\left(\frac{d^{2}}{d \xi^{2}}+\frac{1-4 m^{2}}{4 \xi^{2}}-\mathscr{F} \xi^{4}+2 E \xi^{2}+4 \beta_{1}\right) u(\xi)=0, \\
\left(\frac{d^{2}}{d \eta^{2}}+\frac{1-m^{2}}{4 \eta^{2}}+\frac{\mathscr{F}}{4} \eta+\frac{\beta_{2}}{\eta}+\frac{E}{2}\right) v(\eta)=0,
\end{gathered}
$$

where $m$ is the magnetic quantum number, $E$ is the energy of the electron, and $\beta_{1}$ and $\beta_{2}$ are separation constants related by $\beta_{1}+\beta_{2}=1$. Equations (5) and (6) have eigenvalues $2 \beta_{1}$ and $E / 4$, respectively. The energy $E$ in Eq. (5) is a parameter in the effective potential, rather than a eigenvalue. A procedure for the solution of these equations will be outlined in Sec. III.

To derive the expression for outgoing wave functions, one has to deal with the interaction of $\mathrm{H}$ atoms and the radiation field. Let us turn to the Schrödinger equation with a source,

$$
(E-\widehat{H}) \Psi^{+}(\boldsymbol{r})=D \Psi_{\text {ini }}(\boldsymbol{r}),
$$

where $\Psi^{+}(\boldsymbol{r})$ is the outgoing wave function, $D$ is the dipole operator, and $\Psi_{\mathrm{ini}}(\boldsymbol{r})$ is the wave function for the initial bound state of the system. The radiation field acting on the initial 

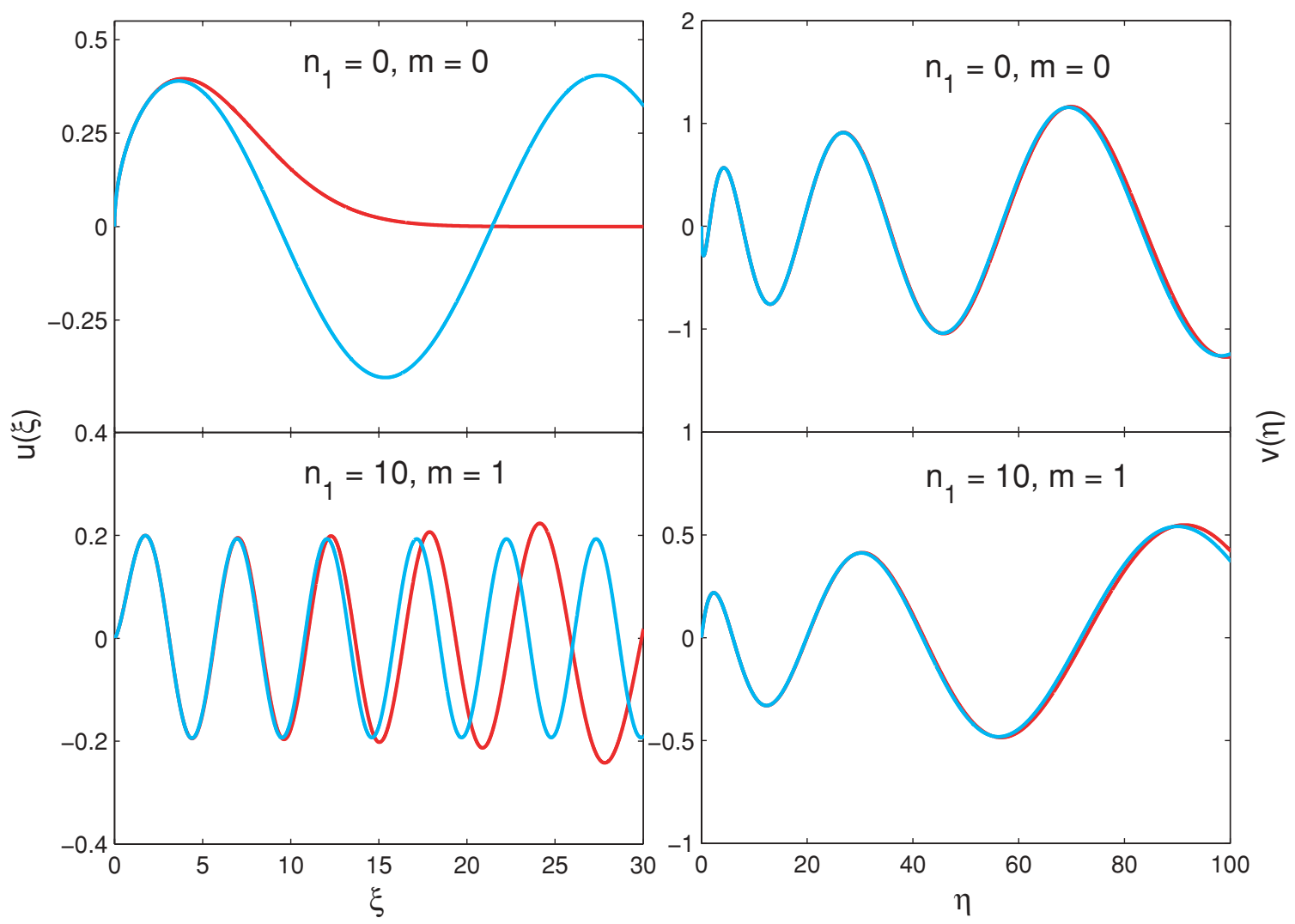

FIG. 1. (Color online) Comparison between numerical solutions (red or dark gray) of the Schrödinger equations and Bessel functions (cyan or light gray). Hydrogen atoms are placed in an electric field $\mathscr{F}=600 \mathrm{~V} / \mathrm{cm}$, and the electron energy is selected to be $E=-120 \mathrm{~cm}^{-1} . u(\xi)$ and $v(\xi)$ specified with the cyan (light gray) curves are equal to, respectively, $\kappa_{u} \sqrt{\xi} J_{m}\left(\sqrt{4 \beta_{1}} \xi\right)$ and $\kappa_{v} \sqrt{\eta} J_{m}\left(2 \sqrt{\beta_{2} \eta}\right)$, where $J_{m}$ denotes the Bessel function, and $\kappa_{u}$ and $\kappa_{v}$ are constants for matching the normalized $u(\xi)$ and energy-normalized $v(\eta)$.

bound state produces the term, which is regarded as a source term in the Schrödinger equation [7,8], on the right-hand side of Eq. (7). The solution $\Psi^{+}(\boldsymbol{r})$ can be expressed as the Green's function $G\left(\boldsymbol{r}, \boldsymbol{r}^{\prime}\right)$, acting upon the source,

$$
\Psi^{+}(\boldsymbol{r})=\int G\left(\boldsymbol{r}, \boldsymbol{r}^{\prime}\right) D \Psi_{\text {ini }}\left(\boldsymbol{r}^{\prime}\right) d \boldsymbol{r}^{\prime} .
$$

The Green's function satisfies

$$
\left[E-\left(-\frac{1}{2} \nabla^{2}-\frac{1}{r}+\mathscr{F} z\right)\right] G\left(\boldsymbol{r}, \boldsymbol{r}^{\prime}\right)=\delta\left(\boldsymbol{r}-\boldsymbol{r}^{\prime}\right) .
$$

Let us expand the three-dimensional Green's function $G\left(\boldsymbol{r}, \boldsymbol{r}^{\prime}\right)$ using the eigenfunctions $\frac{u_{n_{1}}(\xi)}{\sqrt{\xi}}$ and $\frac{e^{i m \phi}}{\sqrt{2 \pi}}$,

$$
G\left(\boldsymbol{r}, \boldsymbol{r}^{\prime}\right)=\sum_{n_{1}, m} \frac{u_{n_{1}}^{*}\left(\xi^{\prime}\right)}{\sqrt{\xi^{\prime}}} \frac{e^{-i m \phi^{\prime}}}{\sqrt{2 \pi}} g_{n_{1}, m}\left(\eta, \eta^{\prime}\right) \frac{u_{n_{1}}(\xi)}{\sqrt{\xi}} \frac{e^{i m \phi}}{\sqrt{2 \pi}},
$$

where $n_{1}$ is the parabolic quantum number specifying the eigenstate. In the mixed parabolic and semiparabolic coordinates, the operator $\nabla^{2}$ and the delta function $\delta\left(\boldsymbol{r}-\boldsymbol{r}^{\prime}\right)$ can be written as

$$
\begin{gathered}
\nabla^{2}=\frac{1}{\xi^{2}+\eta}\left[\frac{1}{\xi} \frac{\partial}{\partial \xi}\left(\xi \frac{\partial}{\partial \xi}\right)+4 \frac{\partial}{\partial \eta}\left(\eta \frac{\partial}{\partial \eta}\right)\right]+\frac{1}{\xi^{2} \eta} \frac{\partial^{2}}{\partial \phi^{2}} \\
\delta\left(\boldsymbol{r}-\boldsymbol{r}^{\prime}\right)=\frac{2}{\xi\left(\xi^{2}+\eta\right)} \delta\left(\xi-\xi^{\prime}\right) \delta\left(\eta-\eta^{\prime}\right) \delta\left(\phi-\phi^{\prime}\right)
\end{gathered}
$$

Substitution of Eqs. (10)-(12) into Eq. (9) and multiplication of the resulting equation by $\frac{\xi^{2}+\eta}{2}$ gives

$$
\begin{aligned}
& \left(\frac{\partial}{\partial \eta} \eta \frac{\partial}{\partial \eta}-\frac{m^{2}}{4 \eta}+\frac{\mathscr{F}}{4} \eta^{2}+\beta_{2}+\frac{E}{2} \eta\right) \\
& \quad \times \sum_{n_{1}, m} u_{n_{1}}^{*}\left(\xi^{\prime}\right) \frac{e^{-i m \phi^{\prime}}}{\sqrt{2 \pi}} g_{n_{1}, m}\left(\eta, \eta^{\prime}\right) u_{n_{1}}(\xi) \frac{e^{i m \phi}}{\sqrt{2 \pi}} \\
& \quad=\delta\left(\xi-\xi^{\prime}\right) \delta\left(\eta-\eta^{\prime}\right) \delta\left(\phi-\phi^{\prime}\right),
\end{aligned}
$$

where Eq. (5) has been used. Multiplying the two sides of this equation by $u_{\bar{n}_{1}}\left(\xi^{\prime}\right) \frac{e^{i \bar{m} \phi^{\prime}}}{\sqrt{2 \pi}}$ and integrating over $\xi^{\prime}, \phi^{\prime}$, we have

$$
\begin{aligned}
& \left(\frac{\partial}{\partial \eta} \eta \frac{\partial}{\partial \eta}-\frac{m^{2}}{4 \eta}+\frac{\mathscr{F}}{4} \eta^{2}+\beta_{2}+\frac{E}{2} \eta\right) g_{n_{1}, m}\left(\eta, \eta^{\prime}\right) \\
& \quad=\delta\left(\eta-\eta^{\prime}\right),
\end{aligned}
$$

where the normalization of $u_{n_{1}}(\xi)$ is assumed, that is,

$$
\int u_{n_{1}}^{*}(\xi) u_{\bar{n}_{1}}(\xi) d \xi=\delta_{n_{1} \bar{n}_{1}}
$$

and the overlines of $\bar{n}_{1}$ and $\bar{m}$ in Eq. (14) are removed after the operation. Defining a modified Green's function for the $\eta$ coordinate,

$$
\frac{\bar{g}_{n_{1}, m}\left(\eta, \eta^{\prime}\right)}{\sqrt{\eta} \sqrt{\eta^{\prime}}}=g_{n_{1}, m}\left(\eta, \eta^{\prime}\right)
$$


we obtain the inhomogeneous differential equation

$$
\begin{aligned}
& \left(\frac{d^{2}}{d \eta^{2}}+\frac{1-m^{2}}{4 \eta^{2}}+\frac{\mathscr{F}}{4} \eta+\frac{\beta_{2}}{\eta}+\frac{E}{2}\right) \bar{g}_{n_{1}, m}\left(\eta, \eta^{\prime}\right) \\
& =\delta\left(\eta-\eta^{\prime}\right) .
\end{aligned}
$$

The solution of the equation is continuous, but its derivative suffers a discontinuity at $\eta=\eta^{\prime}$. Integrating this equation with respect to $\eta$ from $\eta=\eta^{\prime}-\epsilon$ to $\eta=\eta^{\prime}+\epsilon$ yields

$$
\left.\lim _{\epsilon \rightarrow 0} \frac{d \bar{g}_{n_{1}, m}\left(\eta, \eta^{\prime}\right)}{d \eta}\right|_{\eta=\eta^{\prime}-\epsilon} ^{\eta=\eta^{\prime}+\epsilon}=1 .
$$

The boundary conditions require that the Green's function $\bar{g}_{n_{1}, m}\left(\eta, \eta^{\prime}\right)$ behaves as [9]

$$
\bar{g}_{n_{1}, m}\left(\eta, \eta^{\prime}\right) \sim \begin{cases}v^{\mathrm{reg}}(\eta), & \eta \rightarrow 0, \\ v^{\mathrm{out}}(\eta), & \eta \rightarrow \infty\end{cases}
$$

where $v^{\text {out }}(\eta)=v^{\text {reg }}(\eta)+i v^{\text {irreg }}(\eta)$ represents the outgoing wave in the $\eta$ coordinate, and $v^{\text {reg }}(\eta)$ and $v^{\text {irreg }}(\eta)$ are, respectively, regular and irregular solutions of the Schrödinger equation (6). Except in the vicinity of $\eta=\eta^{\prime}$, the Green's function $\bar{g}_{n_{1}, m}\left(\eta, \eta^{\prime}\right)$ should satisfy Eq. (6). This further implies that the form of this Green's function in the $\eta$ coordinate is

$$
\bar{g}_{n_{1}, m}\left(\eta, \eta^{\prime}\right)= \begin{cases}C v^{\text {reg }}(\eta) v^{\text {out }}\left(\eta^{\prime}\right), & \eta \leqslant \eta^{\prime}, \\ C v^{\text {out }}(\eta) v^{\text {reg }}\left(\eta^{\prime}\right), & \eta \leqslant \eta^{\prime},\end{cases}
$$

where $C$ is a constant that can be determined by the discontinuity of the derivative of $\bar{g}_{n_{1}, m}\left(\eta, \eta^{\prime}\right)$ at $\eta=\eta^{\prime}$. Substitution of Eq. (20) into Eq. (18) gives $-C W=1$, where $W$ is the Wronskian for $v^{\text {reg }}\left(\eta^{\prime}\right)$ and $v^{\text {out }}\left(\eta^{\prime}\right)$. It is readily determined to be $W=\frac{i}{2 \pi}$ utilizing Eq. (34). The Green's function in Eq. (20) is further written as

$$
\bar{g}_{n_{1}, m}\left(\eta, \eta^{\prime}\right)=2 \pi i v^{\mathrm{reg}}\left(\eta_{<}\right) v^{\text {out }}\left(\eta_{>}\right),
$$

where $\eta_{>}=\max \left(\eta, \eta^{\prime}\right)$ and $\eta_{<}=\min \left(\eta, \eta^{\prime}\right)$. Inserting this equation into Eq. (10), we have the expression for the three-dimensional Green's function, and as a consequence the outgoing wave function is finally obtained as

$$
\Psi^{+}(\xi, \eta, \phi)=\sum_{n_{1}, m} \frac{u_{n_{1}}(\xi)}{\sqrt{\xi}} \frac{v_{n_{1}}^{\text {out }}(\eta)}{\sqrt{\eta}} \frac{e^{i m \phi}}{\sqrt{2 \pi}} D_{n_{1}, m},
$$

with the dipole transition matrix element

$$
D_{n_{1}, m}=2 \pi i \int \frac{u_{n_{1}}\left(\xi^{\prime}\right)}{\sqrt{\xi^{\prime}}} \frac{v_{n_{1}}^{\mathrm{reg}}\left(\eta^{\prime}\right)}{\sqrt{\eta^{\prime}}} \frac{e^{-i m \phi^{\prime}}}{\sqrt{2 \pi}} D \Psi_{\mathrm{ini}}\left(\xi^{\prime}, \eta^{\prime}, \phi^{\prime}\right) d \boldsymbol{r}^{\prime}
$$

\section{DESCRIPTION OF CALCULATIONS}

According to Eq. (22), regular and irregular solutions of the homogeneous differential equation (6) are needed in order to compute the outgoing wave functions. In the section, we describe how to numerically integrate the Schrödinger equations (5) and (6). The forms of the two equations show that the motion along the $\xi$ coordinate is always finite, while that along the $\eta$ coordinate is oscillatory as $\eta \rightarrow \infty$ [i.e., Eq. (5) is a bound-state eigenvalue problem with the eigenvalue $2 \beta_{1}$, while Eq. (6) is a continuous-state eigenvalue problem with the eigenvalue $\left.\frac{1}{4} E\right]$. Thus $u(\xi)$ and $v(\eta)$ represent, respectively, the eigenfunctions for discrete states and continuum states. The two equations are interrelated by $\beta_{1}+\beta_{2}=1$.

\section{A. Numerical integration of bound-state equations}

We divide the one-dimensional $\xi$ space into two regions, the inner region and outer region. The inner region is a small vicinity near the nucleus, and the outer region is outside the small vicinity up to a sufficiently large value of $\xi$. In the first region, we expand $u(\xi)$ in a power series about the origin $\xi=0[3]$,

$$
u(\xi)=\xi^{|m|+\frac{1}{2}} \sum_{i=0}^{\infty} c_{i} \xi^{i}
$$

where the indexes $i$ are zero and positive even integers, and $c_{i}$ $(i \neq 0)$ are the expansion coefficients of the series, given by a recursion relation

$$
\begin{gathered}
c_{2}=-\frac{\beta_{1} c_{0}}{1+m}, \\
c_{4}=-\frac{2 \beta_{1} c_{2}+E c_{0}}{4(2+m)}, \\
c_{i}=-\frac{4 \beta_{1} c_{i-2}+2 E c_{i-4}-\mathscr{F} c_{i-6}}{i(i+2 m)} \quad(i \geqslant 6) .
\end{gathered}
$$

All $c_{i}$ are expressed in terms of $c_{0}$ using these recurrence relations, while $c_{0}$ is determined by the normalization of $u(\xi)$.

In the outer region, the renormalized Numerov method of Johnson [10] is adopted. Outward integration of Eq. (5) begins from the boundary of the inner region and inward integration is from a large value of $\xi$, denoted with $\xi_{\max }$. For the cases we considered, we could take this value to be $100 \leqslant \xi_{\max } \leqslant 200$. If parabolic instead of semiparabolic coordinates were used, the range of $\xi$ increases to $10^{4}$ rather than $10^{2}$; that would make the integration more time consuming because many more mesh points would be required.

The wave functions for the outward and inward integrations are matched at a suitable point. We first give the node number of the wave function $u(\xi)$ and then the eigenvalue can be found by an iterative procedure. The node number is the parabolic quantum number specifying the eigenstate.

\section{B. Numerical integration of continuous-state equations}

The one-dimensional $\eta$ space is also divided into two regions, the inner region and outer region. In the inner region, a small vicinity near the nucleus, the eigenfunction $v(\eta)$ is expanded in a power series about the origin $\eta=0$ (also see Eq. (10) of Ref. [5]),

$$
v(\eta)=\eta^{\frac{|m|+1}{2}} \sum_{i=0}^{\infty} d_{i} \eta^{i} .
$$

where $d_{i}(i=0,1,2, \ldots)$ are the expansion coefficients of the series, given by

$$
\begin{gathered}
d_{1}=-\frac{\beta_{2} d_{0}}{1-\frac{1}{4} m^{2}}, \\
d_{2}=-\frac{\beta_{2} d_{1}+\frac{1}{2} E d_{0}}{4-\frac{1}{4} m^{2}},
\end{gathered}
$$




$$
d_{i}=-\frac{\beta_{2} d_{i-1}+\frac{1}{2} E d_{i-2}+\frac{1}{4} \mathscr{F} d_{i-3}}{i^{2}-\frac{1}{4} m^{2}} \quad(i \geqslant 3) .
$$

All the coefficients are expressed in terms of $d_{0}$ with these recursion relations, while $d_{0}$ is determined by matching $v(\eta)$ in the inner and outer regions.

The wave function $v(\eta)$ oscillates rapidly for the high excited states, so it is difficult to directly integrate Eq. (6). In the outer region, we first apply an approach in which oscillatory terms of the wave functions are extracted out, as proposed by Milne [11]. Based on the approach of Milne, Eq. (6) is transformed into the nonlinear Milne equation,

$$
\frac{d^{2} \mathcal{M}(\eta)}{d \eta^{2}}=-\omega(\eta) \mathcal{M}(\eta)+\frac{1}{\mathcal{M}^{3}(\eta)},
$$

with

$$
\omega(\eta)=\frac{1-m^{2}}{4 \eta^{2}}+\frac{\mathscr{F}}{4} \eta+\frac{\beta_{2}}{\eta}+\frac{E}{2},
$$

where $\mathcal{M}(\eta)$ is the Milne function. We numerically integrate the Milne equation so as to obtain the Milne function. Finally, the wave functions $v(\eta)$ are expressed as the Milne function times oscillatory terms,

$$
v(\eta)=\left\{\begin{array}{l}
C_{\mathcal{M}} \mathcal{M}(\eta) \sin \left[\varphi(\eta)+\varphi_{0}\right], \\
C_{\mathcal{M}} \mathcal{M}(\eta) \cos \left[\varphi(\eta)+\varphi_{0}\right],
\end{array}\right.
$$

with the asymptotic form at $\eta \rightarrow \infty$

$$
v(\eta) \rightarrow\left\{\begin{array}{l}
\frac{C_{v}}{\left(\eta+\frac{2 E}{\mathscr{F}}\right)^{1 / 4}} \sin \left[\frac{\sqrt{\mathscr{F}}}{3}\left(\eta+\frac{2 E}{\mathscr{F}}\right)^{3 / 2}+\varphi_{0}\right], \\
\frac{C_{v}}{\left(\eta+\frac{2 E}{\mathscr{F}}\right)^{1 / 4}} \cos \left[\frac{\sqrt{\mathscr{F}}}{3}\left(\eta+\frac{2 E}{\mathscr{F}}\right)^{3 / 2}+\varphi_{0}\right],
\end{array}\right.
$$

where $\varphi(\eta)$ satisfies

$$
\frac{d \varphi(\eta)}{d \eta}=\frac{1}{\mathcal{M}^{2}(\eta)},
$$

$\varphi_{0}$ is the initial phase, and $C_{\mathcal{M}}$ and $C_{v}$ are constants determined by the normalization of $v(\eta)$ (see Appendix). The two expressions of Eq. (34) are regular and irregular solutions, respectively [i.e., $v^{\text {reg }}(\eta)$ and $v^{\text {irreg }}(\eta)$ ]. Equation (36) should be jointly solved with the nonlinear Milne equation. Since the Milne function is very smooth, it is possible to take relatively large size mesh points when integrating the Milne equation.

The renormalized Numerov method [10] is applied to numerically solve the Milne equation. The outward integration begins from the boundary between the inner and outer regions, while the inward integration begins from a sufficiently large $\eta$ value. Matching the wave functions from the outward and inward integration determines the initial phase $\varphi_{0}$. Thus, the outgoing waves in the $\eta$ coordinate, $v^{\text {out }}(\eta)$, are constructed.
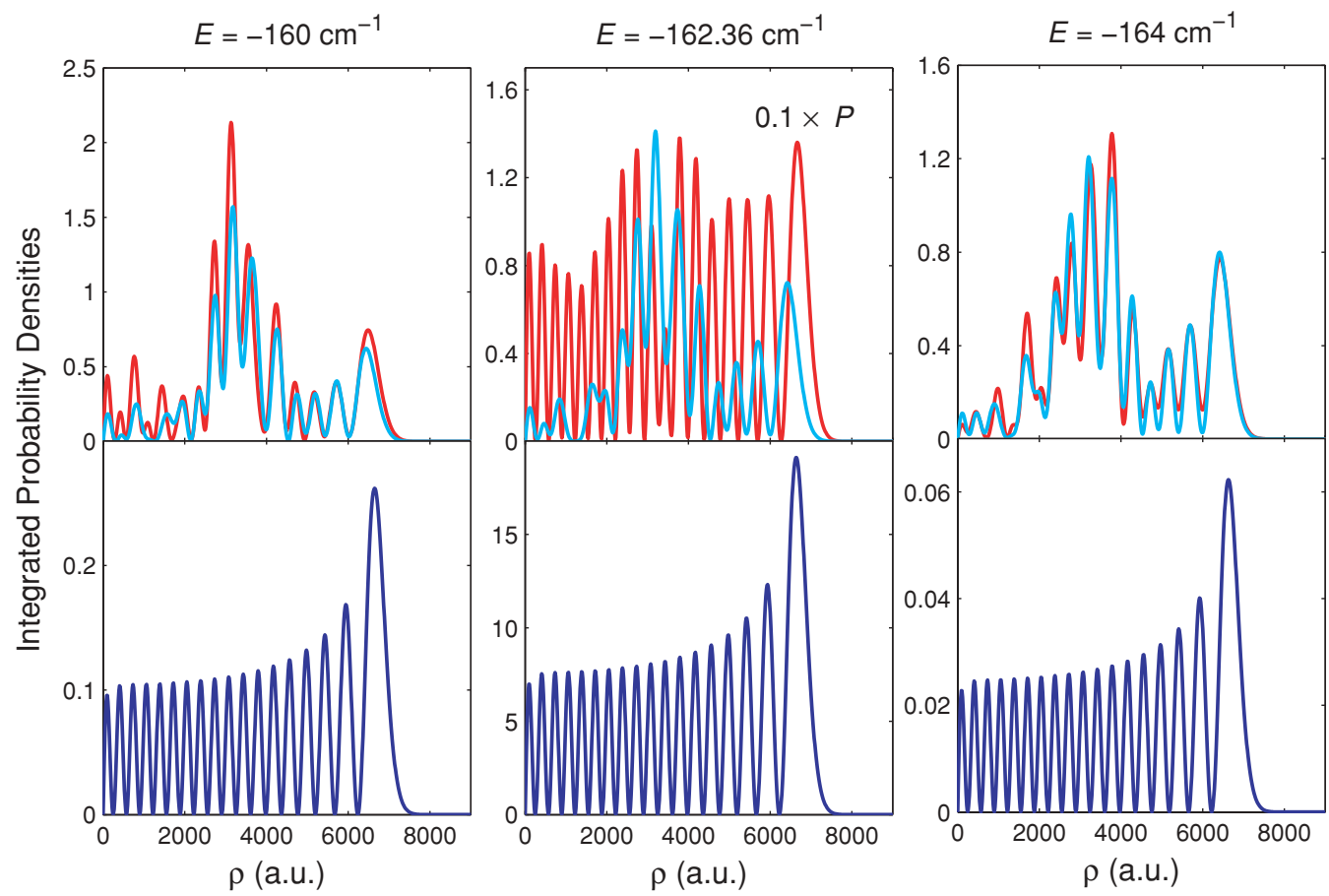

FIG. 2. (Color online) Spatial distributions of probability densities, integrated over the angular variable $\phi$, of the ejected electrons at several electron energies. Hydrogen atoms are placed in an electric field $\mathscr{F}=5714 \mathrm{~V} / \mathrm{cm}$. The detector is placed at $z_{\text {det }}=-1 \mu \mathrm{m}$. The red (dark gray) curves represent the summation in Eq. (22) over all $n_{1}$ until convergence, while the cyan (light gray) curves are the same as the red (dark gray) curves but the contribution from the state $\left(n_{1}, m\right)=(17,0)$ is removed. The curves in the lower panels denote that only the state $(17,0)$ is included. Note the changes in vertical scales, and note that the red (dark gray) curve in the upper central panel is multiplied by 0.1 . The shape and magnitude of the cyan (light gray) curves change only moderately with energy; in contrast, the shape of the blue curves in the lower panels does not change much as the energy passed through the resonance, but the magnitude changes dramatically. 


\section{RESULTS AND DISCUSSION}

In the current calculations, assume that $\mathrm{H}$ atoms in the ground state placed in a uniform electric field are irradiated by a laser light beam with an adjustable frequency. Let the atoms be first excited to $n=2$ states with $m=0$ and then ionized into states near ionization threshold by $\pi$-polarized laser light. For the $n=2$ initial states, the Coulomb potential is much larger than the interaction between the electron and electric field, so the initial state may be calculated with perturbation theory. This is the well-known linear Stark effect. Let us choose the wave function for the initial state $\Psi_{\text {ini }}(\boldsymbol{r})=\frac{1}{\sqrt{2}}\left[\psi_{2 s}(\boldsymbol{r})-\right.$ $\left.\psi_{2 p}(\boldsymbol{r})\right]$, where $\psi_{2 s}(\boldsymbol{r})$ and $\psi_{2 p}(\boldsymbol{r})$ denote wave functions for field-free $\mathrm{H}$ atoms. For the final states, the approach described in the previous section is used to calculate the wave functions.

It is useful to check our numerical solutions by comparison with known analytical results. Let us consider special areas where $\xi$ and $\eta$ are small. In these areas, two terms of the effective potential in Eq. (5), $-\mathscr{F} \xi^{4}$ and $2 E \xi^{2}$, and one term of the effective potential in Eq. (6), $\frac{\mathscr{F}}{4} \eta$, are negligible, and therefore the differential equations (5) and (6) reduce to the Bessel equations [12]. Solutions of the resulting equations are the Bessel functions. Obviously, in the small vicinity of the nucleus, the numerical solutions should be consistent with the known Bessel functions. Figure 1 plots the compared results in an electric field $\mathscr{F}=600 \mathrm{~V} / \mathrm{cm}$ and at the electron energy $E=-120 \mathrm{~cm}^{-1}$. One sees excellent agreement between our numerical solutions (red or dark gray) and the Bessel functions (cyan or light gray) near the origins. This substantiates the reliability of our computation.

To analyze quantum tunneling effects, we calculated the outgoing electron wave functions of $\mathrm{H}$ atoms in an electric field $\mathscr{F}=5714 \mathrm{~V} / \mathrm{cm}$ with electron energies near the resonances. The probability densities, integrated over the angular variable $\phi$, that is, $\mathcal{P}=\int\left|\Psi^{+}\left(\rho, z_{\mathrm{det}}, \phi\right)\right|^{2} \rho d \phi$, are plotted in Fig. 2 at three energies $E=-160,-162.36$, and $-164 \mathrm{~cm}^{-1}$. Striking variations of distributions of the total probability densities (red or dark gray) are observed with energies. The total probability densities at $-162.36 \mathrm{~cm}^{-1}$ are one order of magnitude larger than those at -160 and $-164 \mathrm{~cm}^{-1}$. The discrepancy is attributed to the presence of a narrow resonance $\left(n_{1}, n_{2}, m\right)=(17,3,0)$ with a width of $0.15 \mathrm{~cm}^{-1}$ [13], where $n_{2}$ is a quantum number specifying a resonance state in $v(\eta)$ at $E=-162.36 \mathrm{~cm}^{-1}$. In general, a product state in Eq. (4) is labeled by energy $E$, the number of nodes $n_{1}$ of $u(\xi)$ related to the separation constant $\beta_{1}$, and azimuthal quantum number $m$ [i.e., $\left.\left(E, n_{1}, m\right)\right]$. A similar quantum number $n_{2}$ specifying a continuous state in Eq. (6) is usually undefined. Only at resonance energies is it suitable to define such a quantum number $n_{2}$. Its value is equal to the number of nodes of $v(\eta)$ inside the effective potential barriers [5]. Even so, one may always specify a state using $\left(n_{1}, m\right)$ together with energy $E$.

We compared the integrated probability densities (blue or dark gray) including only the $\left(n_{1}, m\right)=(17,0)$ state at the three energies in the lower panels in Fig. 2. The shape of the probability densities does not change much as the energy passes through the resonance, but the magnitude changes dramatically (up to two orders). To quantitatively see the effect of the resonance state on the total probability densities, we
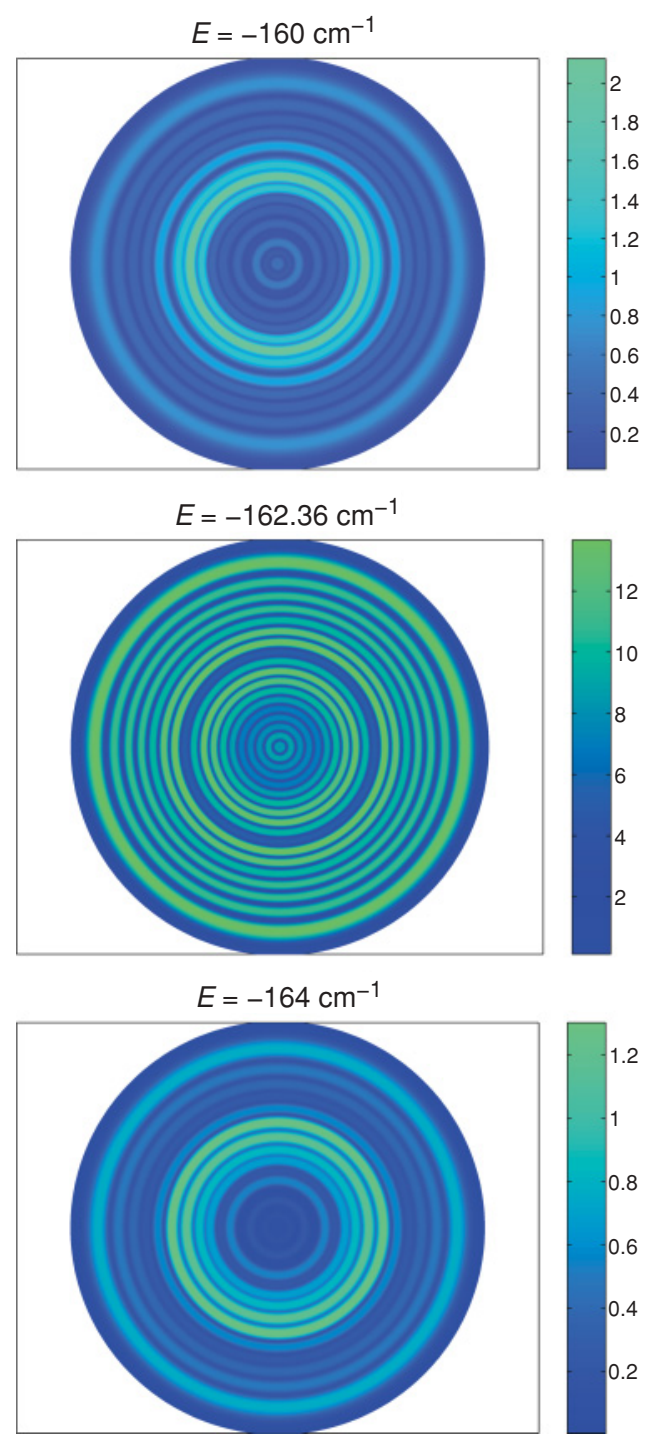

FIG. 3. (Color online) Contour plots of the integrated probability densities corresponding to Fig. 2.

remove the contribution of the $\left(n_{1}, m\right)=(17,0)$ state from the total probability densities and the results are plotted in the same figures (cyan or light gray). It is found that the partial probability densities are of the same order of magnitude and display similar spatial distributions at the three energies. In contrast with the situation at $E=-162.36 \mathrm{~cm}^{-1}$, the $\left(n_{1}, m\right)=(17,0)$ state makes only a small contribution to the total probability densities at $E=-160$ and $-164 \mathrm{~cm}^{-1}$. Contour plots of the probability densities at $E=-160$, -162.36 , and $-164 \mathrm{~cm}^{-1}$ are shown in Fig. 3. One may vividly observe the spatial distributions of probability densities and the strong quantum tunneling effect from these contours.

In the upper panel of Fig. 4, the probability densities from the quantum-mechanical calculations are compared with those from semiclassical open-orbit theory at $E=-100 \mathrm{~cm}^{-1}$. The quantum-mechanical probability densities (orange or wide light gray) are in good agreement with the semiclassical ones (cyan or narrow light gray) except in the regions near the caustics where the semiclassical wave functions are divergent. The uniform approximation (blue or dark gray) has fixed the 


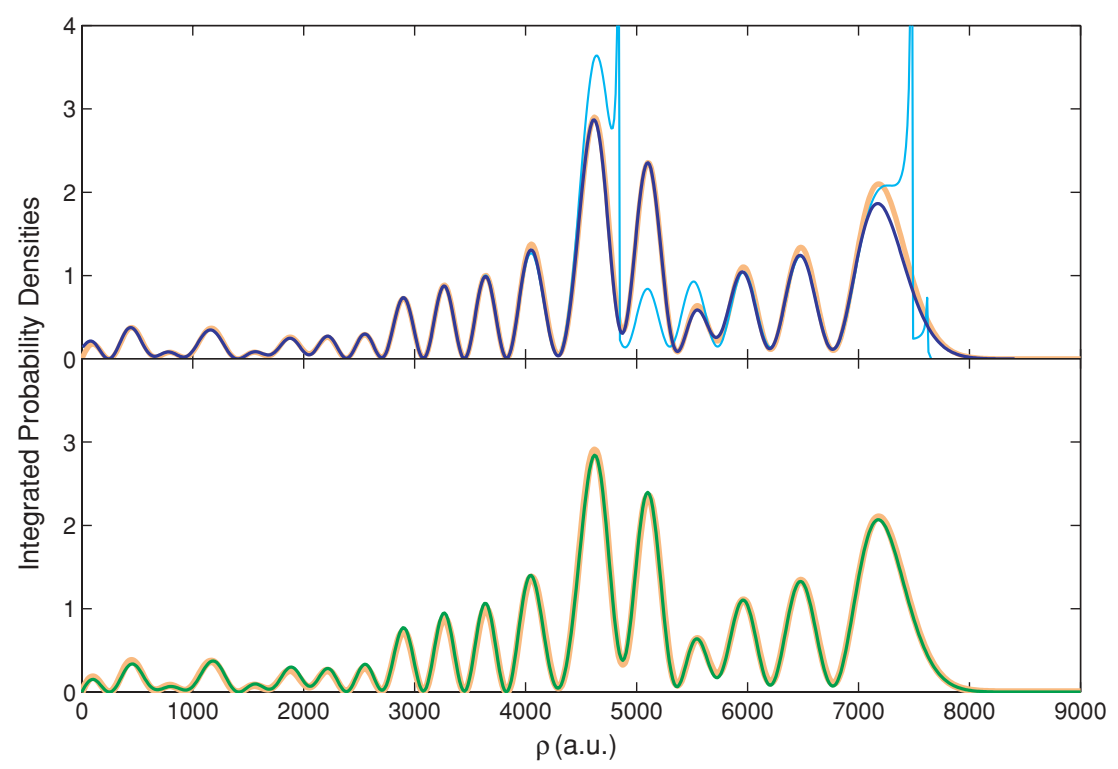

FIG. 4. (Color online) Upper panel: Comparison of integrated probability densities among quantum-mechanical (orange or wide light gray), semiclassical (cyan or narrow light gray), and uniform-approximation (blue or dark gray curve) calculations. The energy of the ejected electron is $E=-100 \mathrm{~cm}^{-1}$, and the electric field is taken to be $\mathscr{F}=5714 \mathrm{~V} / \mathrm{cm}$. The detector is placed at $z_{\mathrm{det}}=-1 \mu \mathrm{m}$. Lower panel: The total (orange or light gray) and partial (green or dark gray) integrated probability densities from the quantum-mechanical calculations.

divergent behaviors of the wave functions near the caustics and excellent agreement is seen between quantum-mechanical and uniform-approximation probability densities. A slight discrepancy between the quantum and uniform-approximation results is visible near $\rho=7200$ a.u. To examine whether quantum tunneling through the potential barrier in $v(\eta)$ gives rise to this discrepancy, total and partial probability densities are compared in the lower panel. Our calculations show that the total probability densities are convergent at $n_{1}=22$, and the height of the potential barrier begins to be larger than the effective energy from $n_{1}=20$. We remove the contribution of the wave functions for $n_{1}=20-22$ from the total probability density and the resulting partial probability density is plotted together with the total probability density in the lower panel. The agreement between the two curves in the lower panel indicates quantum tunneling is small at this energy. The small difference between the quantum and uniform-approximation results in the upper panel appears to provide a measure of the accuracy of open-orbit theory away from resonances.

\section{SUMMARY}

A quantum-mechanical approach has been developed to characterize propagation of electron waves to large distances in photoionization microscopy of hydrogen atoms in uniform external electric fields. We derived the expression for outgoing wave functions from the atomic source due to photoionization and developed a program to numerically solve the Schrödinger equation in mixed parabolic and semiparabolic coordinates. The spatial distributions of probability densities of electron wave propagation, produced by photoionization of hydrogen atoms from the $n=2$ initial states in electric fields, are presented. Comparison has been made with the results from semiclassical open-orbit theory. It is found that open-orbit theory reliably predicts "background" probability densities which vary slowly with energy; resonances, whose positions are easily calculated using the Bohr-Sommerfeld quantization condition described in the preceding paper [1], change spatial distributions of electron probability densities dramatically.

\section{ACKNOWLEDGMENTS}

One of the authors (L.B.Z) would thank Mengli Du for his helpful discussion. This work is supported by NSF.

\section{APPENDIX: NORMALIZATION OF WAVE FUNCTIONS FOR CONTINUOUS STATES $v(\eta)$}

In this appendix, we prove that the asymptotic amplitude of the wave function $v(\eta)$ equals

$$
C_{v}=\frac{1}{\pi^{1 / 2} \mathscr{F} 1 / 4} .
$$

We let $v_{E}(\eta)$ satisfy the energy-normalized condition

$$
\int_{0}^{\infty} v_{E}(\eta) v_{E^{\prime}}(\eta) d \eta=\delta\left(E-E^{\prime}\right)
$$

that is, in a small but finite energy interval $\Delta, v_{E}$ is normalized such that

$$
\begin{aligned}
\int_{\Delta} \int_{0}^{\infty} v_{E}(\eta) v_{E^{\prime}}(\eta) d \eta d E & =\int_{\Delta} \delta\left(E-E^{\prime}\right) d E \\
& = \begin{cases}0, & E^{\prime} \text { outside } \Delta \\
1, & E^{\prime} \text { inside } \Delta .\end{cases}
\end{aligned}
$$

The proof begins from the Schrödinger equation without a source [6],

$$
\left(\frac{d}{d \eta} \eta \frac{d}{d \eta}-\frac{m^{2}}{4 \eta}+\frac{\mathscr{F}}{4} \eta^{2}+\beta_{2}+\frac{E}{2} \eta\right) \frac{v_{E}(\eta)}{\sqrt{\eta}}=0
$$

It is completely equivalent to Eq. (6). Multiplying both sides of Eq. (A4) on the left by $\frac{v_{E^{\prime}}(\eta)}{\sqrt{\eta}}$, we have

$$
\begin{aligned}
& \frac{v_{E^{\prime}}(\eta)}{\sqrt{\eta}} \frac{d}{d \eta} \eta \frac{d}{d \eta} \frac{v_{E}(\eta)}{\sqrt{\eta}}+\left(-\frac{m^{2}}{4 \eta}+\frac{\mathscr{F}}{4} \eta^{2}+\beta_{2}+\frac{E}{2} \eta\right) \\
& \times \frac{v_{E}(\eta) v_{E^{\prime}}(\eta)}{\eta}=0 .
\end{aligned}
$$


By interchanging $E$ and $E^{\prime}$ of this equation, a similar equation can be obtained. Subtracting the resulting equation from Eq. (A5) yields

$$
\begin{aligned}
& \frac{v_{E^{\prime}}(\eta)}{\sqrt{\eta}} \frac{d}{d \eta} \eta \frac{d}{d \eta} \frac{v_{E}(\eta)}{\sqrt{\eta}}-\frac{v_{E}(\eta)}{\sqrt{\eta}} \frac{d}{d \eta} \eta \frac{d}{d \eta} \frac{v_{E^{\prime}}(\eta)}{\sqrt{\eta}} \\
& +\frac{E-E^{\prime}}{2} v_{E}(\eta) v_{E^{\prime}}(\eta)=0 .
\end{aligned}
$$

Integrating this equation over $\eta$ from 0 to $\bar{\eta}$ and letting $\bar{\eta} \rightarrow \infty$ give

$$
\begin{aligned}
\frac{E-E^{\prime}}{2} \mathcal{I}= & \lim _{\bar{\eta} \rightarrow \infty} \int_{0}^{\bar{\eta}} \frac{v_{E}(\eta)}{\sqrt{\eta}} \frac{d}{d \eta} \eta \frac{d}{d \eta} \frac{v_{E^{\prime}}(\eta)}{\sqrt{\eta}} d \eta \\
& -\lim _{\bar{\eta} \rightarrow \infty} \int_{0}^{\bar{\eta}} \frac{v_{E^{\prime}}(\eta)}{\sqrt{\eta}} \frac{d}{d \eta} \eta \frac{d}{d \eta} \frac{v_{E}(\eta)}{\sqrt{\eta}} d \eta,
\end{aligned}
$$

where $\mathcal{I}$ is defined by

$$
\mathcal{I}=\int_{0}^{\infty} v_{E}(\eta) v_{E^{\prime}}(\eta) d \eta
$$

For simplicity, let us use the notation $\mathcal{Q}$ to denote the left-hand side of Eq. (A7). With the aid of integration by parts, we obtain

$$
\begin{aligned}
\mathcal{Q} & =\lim _{\bar{\eta} \rightarrow \infty}\left[\frac{v_{E}(\eta)}{\sqrt{\eta}} \eta \frac{d}{d \eta} \frac{v_{E^{\prime}}(\eta)}{\sqrt{\eta}}-\frac{v_{E^{\prime}}(\eta)}{\sqrt{\eta}} \eta \frac{d}{d \eta} \frac{v_{E}(\eta)}{\sqrt{\eta}}\right]_{0}^{\bar{\eta}} \\
& =\lim _{\bar{\eta} \rightarrow \infty}\left[v_{E}(\eta) \dot{v}_{E^{\prime}}(\eta)-v_{E^{\prime}}(\eta) \dot{v}_{E}(\eta)\right]_{0}^{\bar{\eta}},
\end{aligned}
$$

where $\dot{v}_{E}(\eta)$ represents the derivative of $v_{E}(\eta)$.

This is an important equation for determining $C_{v}$. In the next step of the derivation, we turn to seek the asymptotic form of $v_{E}(\eta)$. As $\eta$ goes to $\infty$, Eq. (6) reduces to the Airy equation

$$
\frac{d^{2} v_{E}(\eta)}{d \eta^{2}}+\left(\frac{\mathscr{F}}{4} \eta+\frac{E}{2}\right) v_{E}(\eta)=0
$$

The asymptotic behavior of its solution, the Airy function, was just the first expression of Eq. (35). Correspondingly, its derivative is

$$
\dot{v}_{E}(\eta)=C_{v}\left(\frac{\sqrt{\mathscr{F}}}{2} \mu^{1 / 4} \cos v-\frac{1}{4} \mu^{-5 / 4} \sin v\right)
$$

with

$$
\begin{gathered}
\mu=\eta+\frac{2 E}{\mathscr{F}}, \\
\nu=\frac{\sqrt{\mathscr{F}}}{3}\left(\eta+\frac{2 E}{\mathscr{F}}\right)^{3 / 2}+\varphi_{0} .
\end{gathered}
$$

Replacing $E$ in $v_{E}(\eta)$ and $\dot{v}_{E}(\eta)$ by $E^{\prime}$, we obtain two similar equations. Substituting these equations into (A9) gives

$$
\begin{aligned}
\mathcal{Q} & =\lim _{\bar{\eta} \rightarrow \infty} C_{v}^{2} \frac{\sqrt{\mathscr{F}}}{2} \sin \left(v-v^{\prime}\right) \\
& =\lim _{\bar{\eta} \rightarrow \infty} C_{v}^{2} \frac{\sqrt{\mathscr{F}}}{2} \sin \left(\frac{E-E^{\prime}}{\sqrt{\mathscr{F}}} \sqrt{\bar{\eta}}\right),
\end{aligned}
$$

where the boundary condition $v_{E}(\eta=0)=0$ is used, and $v$ is expanded into a Taylor series, in which only the first two terms are kept. Combining Eqs. (A8) and (A14), we have

$$
\mathcal{I}=\lim _{\bar{\eta} \rightarrow \infty} C_{v}^{2} \sqrt{\mathscr{F}} \frac{\sin \left(\frac{E-E^{\prime}}{\sqrt{\mathscr{F}}} \sqrt{\bar{\eta}}\right)}{E-E^{\prime}} .
$$

Integrating Eq. (A15) over $E$ yields

$$
\int_{\Delta} \mathcal{I} d E=\lim _{\bar{\eta} \rightarrow \infty} C_{v}^{2} \sqrt{\mathscr{F}} \int_{\Delta} \frac{\sin \left(\frac{E-E^{\prime}}{\sqrt{\mathscr{F}}} \sqrt{\bar{\eta}}\right)}{E-E^{\prime}} d E=C_{v}^{2} \sqrt{\mathscr{F}} \pi .
$$

Noticing Eq. (A3), we finally obtain the asymptotic amplitude of the wave function $v_{E}(\eta)$ [i.e., Eq. (A1)].
[1] L. B. Zhao and J. B. Delos, Phys. Rev. A 81, 053417 (2010).

[2] L. D. Landau and E. M. Lifshitz, Quantum Mechanics: Non-Relativistic Theory, 3rd ed. (Elsevier, Amsterdam, 1981), Vol. 3.

[3] R. J. Damburg and V. V. Kolosov, J. Phys. B 9, 3149 (1976). The power $\frac{1}{2}$ of $\mu$ in Eq. (11) of this reference is missing. V. V. Kolosov, ibid. 22, 833 (1989).

[4] E. Luc-Koenig and A. Bachelier, J. Phys. B 13, 1743 (1980).

[5] A. Alijah, J. T. Broad, and J. Hinze, J. Phys. B 19, 2617 (1986); A. Alijah, ibid. 25, 5043 (1992).

[6] H. A. Bethe and E. E. Salpeter, Quantum Mechanics of Oneand Two-Electron Atoms (Plenum, New York, 1977), p. 229.
[7] M. L. Du and J. B. Delos, Phys. Rev. A 38, 1896 (1988); 38, 1913 (1988).

[8] M. R. Haggerty and J. B. Delos, Phys. Rev. A 61, 053406 (2000).

[9] L. S. Rodberg and R. M. Thaler, Introduction to the Quantum Theory of Scattering (Academic, New York, 1967), p. 82.

[10] B. R. Johnson, J. Chem. Phys. 67, 4086 (1977).

[11] W. E. Milne, Phys. Rev. 35, 863 (1930).

[12] M. Abramowitz and I. E. Stegun eds., Handbook of Mathematical Functions (Department of Commerce, Washington, DC, 1972), entries (9.1.49) and (9.1.50).

[13] G. Alvarez, R. J. Damburg, and H. J. Silverstone, Phys. Rev. A 44, 3060 (1991). 